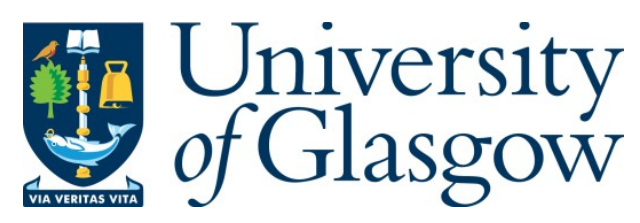

Mohamed, A., Onireti, O., Hoseinitabatabaei, S. A., Imran, M., Imran, A., and Tafazolli, R. (2015) Mobility Prediction for Handover Management in Cellular Networks with Control/Data Separation. In: 2015 IEEE International Conference on Communications (ICC), London, UK, 08-12 Jun 2015, pp. 3939-3944. ISBN 9781467364324.

There may be differences between this version and the published version. You are advised to consult the publisher's version if you wish to cite from it.

http://eprints.gla.ac.uk/133430/

Deposited on: 4 January 2017

Enlighten - Research publications by members of the University of Glasgow http://eprints.gla.ac.uk 


\title{
Mobility Prediction for Handover Management in Cellular Networks with Control/Data Separation
}

\author{
Abdelrahim Mohamed*, Oluwakayode Onireti*, Seyed Amir Hoseinitabatabaei*, \\ Muhammad Imran*, Ali Imran ${ }^{\dagger}$, Rahim Tafazolli* \\ * Institute for Communications Systems ICS, University of Surrey, Guildford, UK \\ ${ }^{\dagger}$ School of Electrical and Computer Engineering, University of Oklahoma, Tulsa, USA \\ E-mail: abdelrahim.mohamed @ surrey.ac.uk
}

\begin{abstract}
In research community, a new radio access network architecture with a logical separation between control plane (CP) and data plane (DP) has been proposed for future cellular systems. It aims to overcome limitations of the conventional architecture by providing high data rate services under the umbrella of a coverage layer in a dual connection mode. This configuration could provide significant savings in signalling overhead. In particular, mobility robustness with minimal handover (HO) signalling is considered as one of the most promising benefits of this architecture. However, the DP mobility remains an issue that needs to be investigated. We consider predictive DP HO management as a solution that could minimise the out-ofband signalling related to the HO procedure. Thus we propose a mobility prediction scheme based on Markov Chains. The developed model predicts the user's trajectory in terms of a HO sequence in order to minimise the interruption time and the associated signalling when the $\mathrm{HO}$ is triggered. Depending on the prediction accuracy, numerical results show that the predictive HO management strategy could significantly reduce the signalling cost as compared with the conventional nonpredictive mechanism.
\end{abstract}

Index Terms-Base station; cellular systems; control data separation architecture; handover; LTE; Markov Chain; mobility history; mobility prediction.

\section{INTRODUCTION}

Wireless data traffic is increasing dramatically due to proliferation of smart devices and the high dependency on mobile communications in everyday life. Among the possible techniques to overcome the capacity crunch problem, network densification is seen as the most promising solution [1], [2]. It has been estimated that 50 million base stations (BSs) will be deployed by 2020 [3]. Although these estimations are debatable, they give an indication of the situation in the near future. Such massive deployments raise several problems in terms of energy consumption, mobility management, capital and running costs, planning and scalability. Most of these issues are tightly coupled to the radio access network (RAN) architecture which constitutes an integral part of cellular systems.

With ultra-dense small cell deployments, mobility management becomes complex because handovers (HOs) will happen frequently even for low mobility users. In the conventional cellular architecture, the $\mathrm{HO}$ procedure includes transferring all channels (i.e., control and data) from one BS to another with a significant signalling load [4]. With a frequent $\mathrm{HO}$ rate, the signalling overhead and the call drop rates could increase dramatically. Recently, a futuristic RAN architecture with a logical separation between control plane (CP) and data plane (DP) has been proposed to resolve these issues [5]-[7]. In the control/data separation architecture (CDSA), a few macro cells, known as control base stations (CBSs), provide the basic connectivity services and support efficient control signalling. Within the CBS footprint, on-demand high data rate services are provided by dedicated small cells known as data base stations (DBSs). As shown conceptually in Fig. 1, all user equipments (UEs) are anchored to the CBS, while the active UEs are associated with both the CBS and the DBS in a dual connection mode [5].

This configuration could offer simple and robust HO procedures because the radio resource control (RRC) connection is maintained by the CBS (which is typically a macro cell), hence the UE is anchored to a BS with a large coverage area. As a result, the intra-CBS HOs (i.e., between DBSs under the footprint of the same CBS) do not require changing the control channel, which in turn minimises the $\mathrm{CP} \mathrm{HO}$ failure rate [7], [8]. Nonetheless, a DP $\mathrm{HO}$ is always required when the UE moves from one DBS to another. Given the small coverage area of the DBS, the DP HO signalling could increase dramatically. Context information such as mobility history can play a key role in optimising the RRC and the DP HO process. It can be used to select the most appropriate DBS for a moving terminal, e.g., a DBS with the highest probability that the user will not leave it quickly [6], [9]. In addition, predicting the DBSs that the UE will visit allows these DBSs to prepare and reserve resources in advance. This in turn could relax the DP HO requirements and minimise the associated signalling and interruption time [9]. However, such techniques require a reliable mobility prediction scheme.

In this paper, we develop a mobility prediction model for predictive HO management in CDSA networks. A general learning and prediction scheme that is not restricted to a particular scenario is developed, and we evaluate the signalling cost in both predictive and non-predictive HO management strategies. The remainder of this paper is structured as follows: Section II develops the mobility learning and prediction model. Section III discusses the HO procedure with and without mobility prediction and formulates the signalling cost in both cases. Section IV provides numerical results that assess the 


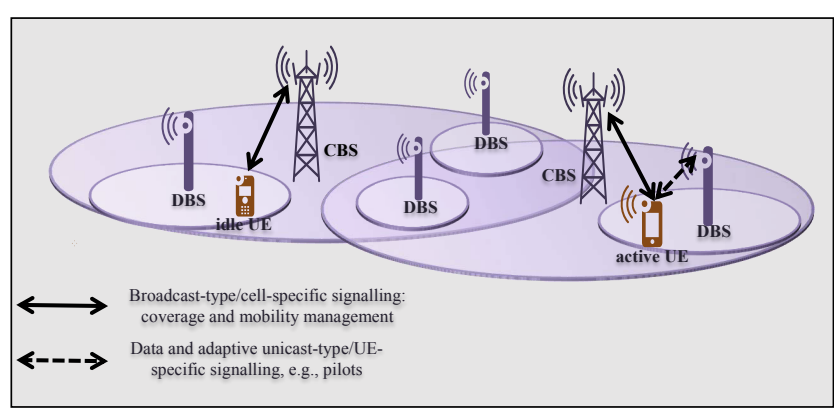

Fig. 1. Control/Data separation architecture

accuracy of the proposed scheme as well as the associated signalling cost. Finally, Section V concludes the paper.

\section{Mobility Prediction Model}

The proposed scheme relies on representing the DP network (i.e., the DBSs) by a discrete-time Markov Chain (DTMC). The latter is a stochastic process characterised by a state space, a transition matrix and an initial distribution [10]. Several variations of the DTMC are used to model and to predict users' mobility, see for example [11]-[14]. Given the problem under study, a $\mathrm{HO}$ from a DBS to another is equivalent to a state transition. Thus each state in the DTMC represents a DBS. Fig. 2 shows a graphical representation of a DTMC with $t_{i, j}$ being the probability of a direct transition (i.e., HO) from $\mathrm{DBS}_{i}$ to $\mathrm{DBS}_{j}$.

The memoryless property of the DTMC implies that the transition matrix would have a static realisation independent of the user's history. In contrast, the proposed model considers a learning transition matrix that can be updated dynamically. Following the derivations of the standard DTMC, the probability distribution can be written as [10]:

$$
\mathbf{p}_{k}=\mathbf{p}_{0} \mathbf{T}^{k}
$$

with

$$
\begin{aligned}
& \mathbf{p}_{k}=\left[\begin{array}{lllll}
p_{1} & p_{2} & p_{3} & \ldots & p_{n}
\end{array}\right] \\
& \mathbf{p}_{0}=\left[\begin{array}{lllll}
\gamma_{1} & \gamma_{2} & \gamma_{3} & \ldots & \gamma_{n}
\end{array}\right] \\
& \mathbf{T}=\left[\begin{array}{cccc}
t_{1,1} & t_{1,2} & \cdots & t_{1, n} \\
t_{2,1} & t_{2,2} & \cdots & t_{2, n} \\
\vdots & \vdots & \vdots & \vdots \\
t_{n, 1} & t_{n, 2} & \cdots & t_{n, n}
\end{array}\right]
\end{aligned}
$$

where $\mathbf{p}_{k}$ is the $k^{\text {th }}$ HO probability vector, i.e., $p_{i}$ is the probability of being at $\mathrm{DBS}_{i}$ after $k$ HOs. $\mathbf{T}$ is the transition probability matrix while $\mathbf{p}_{0}$ is the initial distribution vector. Equation (1) can be used to predict a DBS sequence in the user's path. The prediction depends on mobility history which is reflected by $\mathbf{T}$. In the following, we describe the procedure for updating the transition matrix.

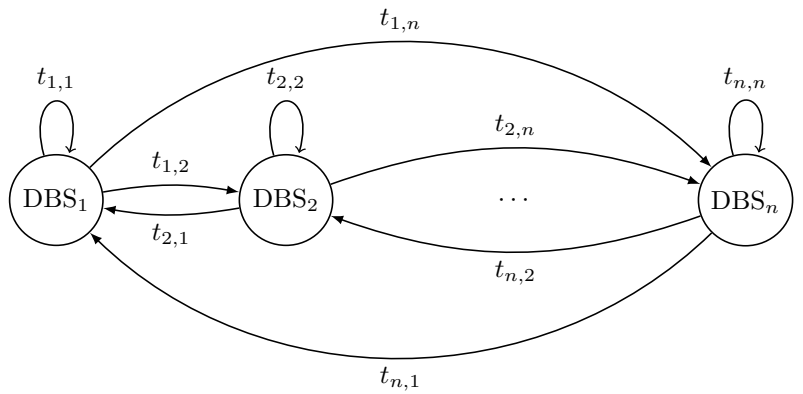

Fig. 2. Discrete-time Markov Chain with $n$ states (i.e., DBSs), only states 1, 2 and $n$ are shown for readability

\section{A. Transition Matrix: Properties and Conditions}

Consider $\mathbb{S}$ as the DTMC state space with $\mathbb{I}$ being the states' indices. Define $\mathbb{N}_{i}$ as a list of the DBSs that are neighbours ${ }^{1}$ to $\mathrm{DBS}_{i} \forall i \in \mathbb{I}$. Notice that $\mathbb{N}_{i}$ is not a UE-specific parameter, but rather it is a system parameter. The following properties govern $\mathbf{T}$ in the context of the considered transitions (i.e., cellular HOs). These properties are used to set necessary conditions aligned with realistic assumptions.

- Since the number of the DBSs is finite, the DTMC state space is finite:

$$
\mathbb{S}=\left\{\mathrm{DBS}_{1}, \mathrm{DBS}_{2}, \ldots, \mathrm{DBS}_{n}\right\}, \mathbb{I}=\{1,2, \ldots, n\}
$$

- $t_{i, j}$ is a positive real number between 0 and 1 (inclusive):

$$
0 \leq t_{i, j} \leq 1 \quad, \quad \forall i, j \in \mathbb{I}
$$

- A HO from a DBS to itself is not possible (i.e., a pingpong $\mathrm{HO}$ is considered as two HOs between the involved DBSs). Thus $\mathbf{T}$ is a hollow matrix:

$$
t_{i, i}=0 \quad, \quad \forall i \in \mathbb{I}
$$

- The direct HOs are possible between neighbouring DBSs only:

$$
t_{i, j}=t_{j, i}=0 \quad, \quad \forall j \notin \mathbb{N}_{i}
$$

- Any new movement starts from the destination of the previous trajectory. Thus the UE will definitely make an outbound $^{2}$ HO from any DBS. However, the UE may not necessarily perform an inbound HO to all the DBSs in the network. As a result, $\mathbf{T}$ is a right stochastic matrix but not necessarily a doubly stochastic matrix. This property sets the following condition:

$$
\sum_{j=1}^{n} t_{i, j}=1 \quad, \quad \forall i \in \mathbb{I} .
$$

\footnotetext{
${ }^{1}$ The first tier neighbours that can be reached directly in a single HO.

${ }^{2}$ The HO from X to Y is an outbound HO from X's point of view and it is an inbound $\mathrm{HO}$ from Y's perspective.
} 


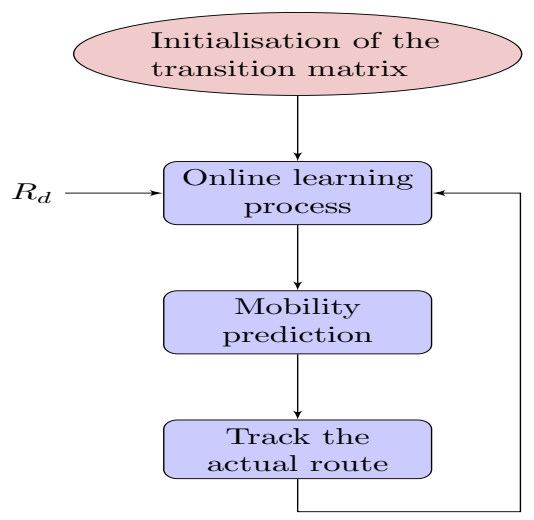

Fig. 3. Block diagram of the proposed mobility learning and prediction scheme

\section{B. Transition Matrix Initialisation}

The mobility prediction entity (MPE) constructs a $n \times n$ transition matrix for each user and initialises it according to the conditions of Section II-A. The process of initialising $\mathbf{T}$ involves invoking conditions (4) and (5) to ensure a zero probability for the direct HOs from a DBS to itself or to a non-neighbouring DBS, respectively. Then the remaining elements in $\mathbf{T}$ are initialised with an equi-probable outbound HO assumption, since the new users do not have a mobility history. Algorithm 1 illustrates the initialisation procedure.

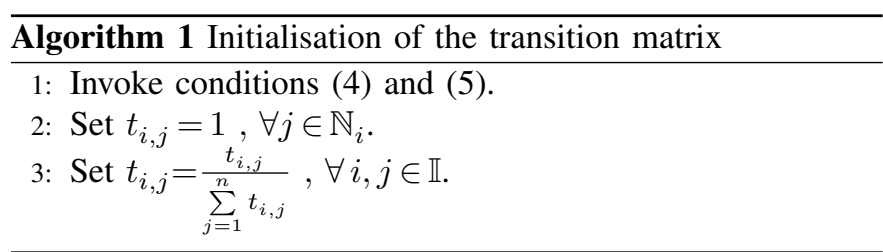

\section{Online Learning Process}

The MPE tracks the user's movement (in terms of HOs) and updates $\mathbf{T}$ accordingly. Fig. 3 shows a block diagram of the proposed mobility learning and prediction scheme. The basic idea is to favour the most common routes followed by the user by giving them higher probabilities compared with other routes. A recent trajectory dependency parameter, $R_{d}$, where $0 \leq R_{d} \leq 1$ is proposed to control the model's reaction to random or less frequent movements. Small (large) values of $R_{d}$ indicate that the MPE has a low (high) confidence in the regularity of the user, hence each trajectory will have a low (high) impact on the updated $\mathbf{T}$. The extreme case of $R_{d}=0$ means that $\mathbf{T}$ will not be updated (hence the prediction is independent of the movement history), while the case of $R_{d}=1$ biases the prediction towards the most recent trajectory.

The process of updating $\mathbf{T}$ can be described by the following example without loss of generalisation. Suppose a user following the path: $\mathrm{DBS}_{a} \rightarrow \mathrm{DBS}_{b} \rightarrow \mathrm{DBS}_{c}$. Then for each $\mathrm{HO}$ e.g., from $\mathrm{DBS}_{a}$ to $\mathrm{DBS}_{b}$, the MPE updates the probabilities of outbound HOs from $\mathrm{DBS}_{a}$ to each neighbouring DBS in a game scheme of several stages. In the first stage, $\mathrm{DBS}_{b}$ and the subset of the DBSs in $\mathbb{N}_{a}$ that have non-zero probabilities for inbound $\mathrm{HOs}$ from $\mathrm{DBS}_{a}$ participate in the game. i.e.,

$$
\mathbb{P S}_{1}=\left\{\mathrm{DBS}_{j}: j \in \mathbb{N}_{a} \wedge t_{a, j}>0\right\} \cup\left\{\mathrm{DBS}_{b}\right\},
$$

where $\mathbb{P S}_{x}$ is the players set in stage $x \geq 1$ of the game. In the first stage, the probability of the direct $\mathrm{HO}$ from $\mathrm{DBS}_{a}$ towards $\mathrm{DBS}_{b}$ is increased by a certain amount controlled by $R_{d}$. Similarly, the probabilities of the direct HOs from $\mathrm{DBS}_{a}$ towards all other playing DBSs (i.e., except $\mathrm{DBS}_{b}$ ) are decreased. This can be expressed mathematically as:

$$
t_{a, b}^{(1)}=t_{a, b}+\sum_{j} t_{a, j} R_{d} \quad, \quad \forall \mathrm{DBS}_{j} \in \mathbb{P S}_{1} \backslash\left\{\mathrm{DBS}_{b}\right\}
$$

$$
t_{a, j}^{(1)}=t_{a, j}-\frac{\sum_{j} t_{a, j} R_{d}}{\left|\mathbb{P S}_{1}\right|-1} \quad, \quad \forall \mathrm{DBS}_{j} \in \mathbb{P S}_{1} \backslash\left\{\mathrm{DBS}_{b}\right\}
$$

where $\left|\mathbb{P S}_{x}\right|$ is the cardinality of the set $\mathbb{P S}_{x}$, the superscript $(x)$ means the probability after stage $x$. It can be noticed that the first stage may violate condition (3) because $t_{a, b}$ and $t_{a, j}$ are increased and decreased, respectively, without bounds. A simple solution would be setting a lower bound of 0 and an upper bound of 1 for each entry in $\mathbf{T}$. However this may lead to violating condition (6) because the amount of increase and decrease in the probabilities may not be the same in some cases.

To solve this problem, additional stages are added to reach an equilibrium without violating the conditions of Section II-A or affecting the learned history. In stage $x>1$, the DBSs with zero or negative probabilities after stage $x-1$ leave the game. The DBSs with positive probabilities are called survivals and they equally share the negative probabilities resulted from stage $x-1$. In other words, the player set in stage $x>1$ includes the survivals only, i.e.,

$$
\mathbb{P S}_{x}=\left\{\operatorname{DBS}_{j}: t_{a, j}^{(x-1)}>0 \wedge \mathrm{DBS}_{j} \in \mathbb{P S}_{x-1}\right\} .
$$

Since the survivals share the negative entries, their probabilities are equally decreased as:

$$
t_{a, j}^{(x)}=t_{a, j}^{(x-1)}+\frac{\sum_{n} t_{a, n}^{(x-1)}}{\left|\mathbb{P S}_{x}\right|}, \forall \mathrm{DBS}_{j} \in \mathbb{P S}_{x},
$$

where $x>1, \mathrm{DBS}_{n} \in \mathbb{P S}_{x-1}$ and $t_{a, n}^{(x-1)}<0$. Notice that the second term of (11) is negative (i.e., the summation in (11) is for the negative probabilities that resulted from stage $x-1)$. The MPE adds several consecutive stages until all the entries in $\mathbf{T}$ are not negative. Once $\mathbf{T}$ is updated (i.e., after the final stage), the user's trajectory can be predicted by using (1). Given a source DBS where the user starts its current movement, a sequence of candidate DBSs in the user's path can be predicted according to the user's history (which is reflected by $\mathbf{T}$ ). This can be done by invoking (1) with $k=1,2,3, \ldots$ and $\gamma_{i}=1$ for the source DBS, and then selecting the DBS with the highest probability in each HO i.e.,

$$
k^{\text {th }} \mathrm{HO} \mathrm{DBS}=\left.\mathrm{DBS}_{w}\right|_{p_{w}=\max \left(\mathbf{p}_{k}\right)} .
$$




\section{Handover Procedure And Cost}

As discussed in Section I, a reliable prediction of the user's trajectory allows the candidate DBSs to prepare and reserve resources in advance, which in turn could simplify the HO process and minimise the associated overhead and interruption time. To investigate this claim, we consider the typical long term evolution (LTE) X2 HO procedure as a benchmark for the non-predictive HO scenario. In the latter, the UE measures signals of the detectable $\mathrm{DBSs}^{3}$ and reports the result to the serving (i.e., the source) DBS whenever the HO criteria is met. The HO procedure consists of three major steps: preparation, execution and completion. In the preparation phase, the source DBS determines the target DBS and establishes a connection with it via the X2 interface. Then the target DBS performs an admission control, reserves resources for the UE and some parameters related to the UE security and ciphering are exchanged between the source and the target DBSs. In the execution phase, the UE detaches from the source DBS and accesses the target DBS. Finally, the HO completion phase switches the DP path towards the target DBS [15].

In the predictive $\mathrm{HO}$ procedure, most of the $\mathrm{HO}$ preparation steps can be done before the $\mathrm{HO}$ criteria is met, provided that the prediction is accurate enough. In this case, the predicted DBS can reserve resources for the UE in advance. Similarly, all the necessary parameters can be exchanged between the source and the predicted DBSs before the HO criteria is met (i.e., advance HO preparation). When the UE sends the measurement report indicating that a $\mathrm{HO}$ is required, the source DBS evaluates this report. If the target DBS reported by the UE is the same as the predicted DBS (i.e., correct prediction), then the $\mathrm{HO}$ process proceeds with the execution phase. If the prediction is incorrect (i.e., the predicted DBS is not the target DBS being reported by the UE), then the conventional non-predictive $\mathrm{HO}$ procedure is triggered. In the latter case, an additional signalling is required to cancel the resources that are reserved in the predicted DBS. Fig. 4 shows the signalling flow diagram for these cases.

The HO signalling cost can be expressed in terms of the delay required to transmit and process the HO messages [16]. Denote $\alpha_{i, j}$ as the one way transmission cost between nodes $i$ and $j, \beta_{i}$ as the processing cost in node $i$. The HO signalling cost $C$ can be written as [17]:

$$
C=\sum \alpha_{i, j}+\sum \beta_{i}
$$

Since the actual HO procedure starts after the source DBS receives the measurement report, the $\mathrm{HO}$ signalling cost includes the $\mathrm{HO}$ decision and the subsequent steps (depending on the HO type). In other words, the advance preparation procedure (i.e., steps a, b and c of Fig. 4) is not included in the cost function of the predictive $\mathrm{HO}$ case because the advance reservation phase is completed before the HO is triggered, hence its timing and delay requirements are not critical. The expected cost of the predictive $\mathrm{HO} C_{\text {pred }}$ can be written as:

$$
C_{\text {pred }}=A_{p} C_{\text {corr }}+\left(1-A_{p}\right) C_{\text {incorr }},
$$

\footnotetext{
3 evolved node-B (eNB) in LTE terminology
}

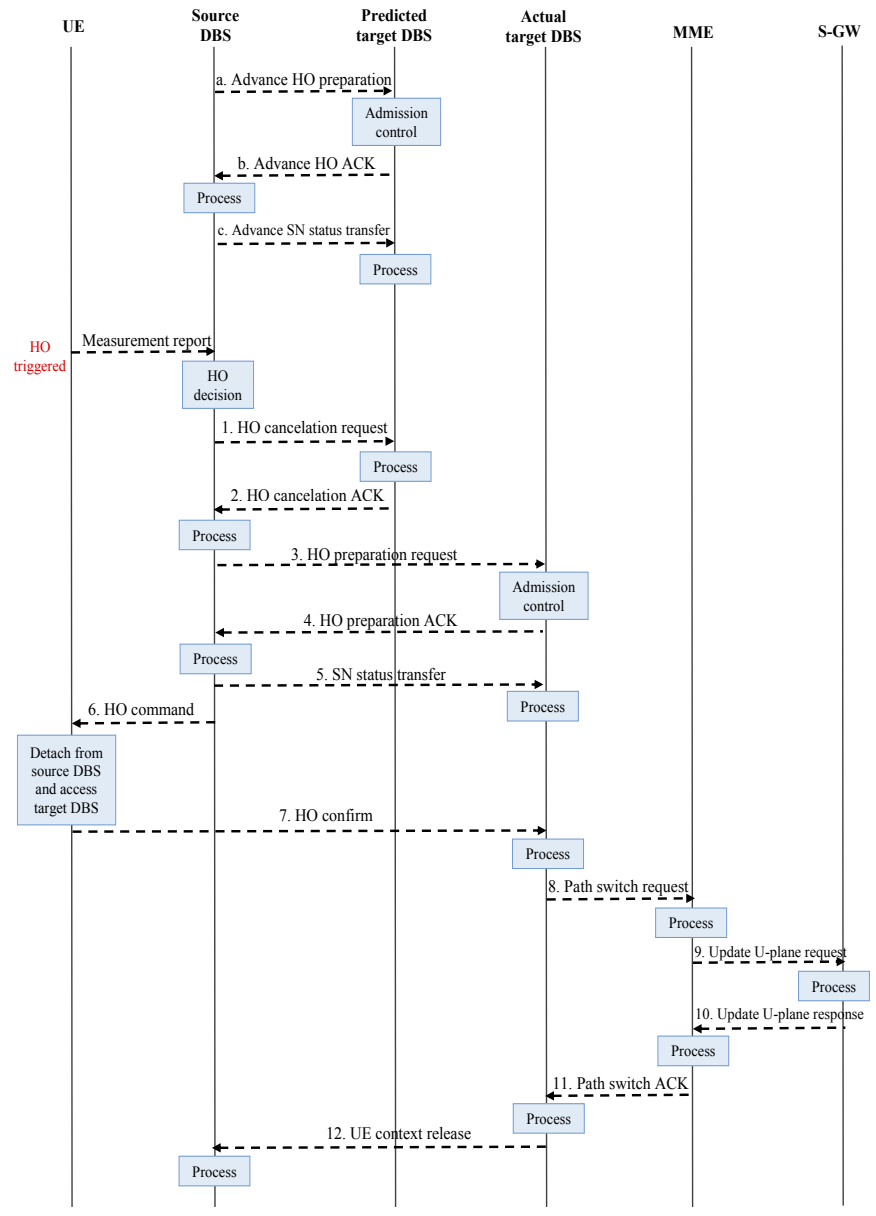

Fig. 4. Signalling flow diagram for predictive and non-predictive $\mathrm{HO}$ scenarios, based on the LTE X 2 HO procedure. Signalling messages in nonpredictive HO: $3,4,5,6,7,8,9,10,11,12$. Signalling messages in predictive HO with correct prediction: $6,7,8,9,10,11,12$. Signalling messages in predictive $\mathrm{HO}$ with incorrect prediction: 1, 2, 3, 4, 5, 6, 7, 8, 9, 10, 11, 12 . Acronym ACK: Acknowledgement, SN: Sequence Number.

where $A_{p}$ is the prediction accuracy, $C_{c o r r}$ and $C_{\text {incorr }}$ are the $\mathrm{HO}$ costs with correct and incorrect predictions, respectively, which can be calculated by (13) in conjunction with Fig. 4.

Although the main parameter analysed in this paper is the HO signalling cost, other system parameters such as the capacity can be affected by the prediction accuracy. For instance, an incorrect prediction may degrade the overall system capacity, since it reserves resource which could otherwise be used for other users. In addition, the time dimension may have an impact on the overall performance. For example, a too early reservation, even with a correct prediction, wastes the system resources because they are reserved for a long time without being used. However, these aspects are left to a separate study.

\section{Performance Evaluation}

\section{A. Mobility prediction accuracy}

HO traces have been generated to assess the accuracy of the proposed mobility prediction scheme. The considered network topology consists of 69 hexagonal shaped DBSs. Traces for 100 consecutive days are generated where the trajecotry of 


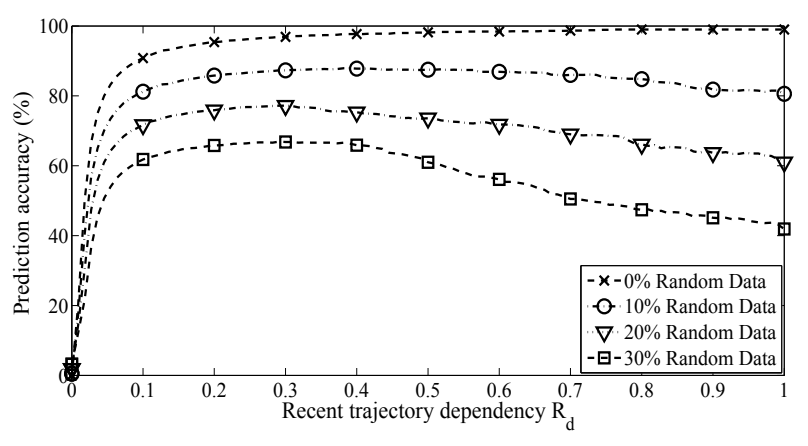

Fig. 5. Prediction accuracy of the proposed mobility prediction model

each day consists of 10 HOs. We consider a regular user that follows the same route every day (i.e., the HO traces have $0 \%$ random data), and a users that follows a regular route in some days and random routes in other days. The percentages of the random days w.r.t. the total period are 10\%, $20 \%$ and $30 \%$ and they are distributed evenly across the 100 day period. The prediction of each day's trajectory is based on the history learned up to the previous day. The evaluation is based on the prediction accuracy which is defined as the ratio between the number of correctly predicted DBSs and the total number of the DBSs visited by the UE, where a correct prediction is triggered only if the predicted DBS is the same as the DBS visited by the UE and in the same order.

Fig. 5 provides the average prediction accuracy for different values of $R_{d}$. In the regular movement scenario (i.e., $0 \%$ random data), the proposed scheme predicts the trajectory with an accuracy of $97-99 \%$ for $R_{d} \geq 0.3$. However, the prediction accuracy decreases at smaller values of $R_{d}$ to reach $92 \%$ with $R_{d}=0.1$. This can be traced to the fact that small $R_{d}$ values require more observations (i.e., a longer history) before a reliable decision can be made. It can be noticed that the prediction accuracy is roughly $0 \%$ when $R_{d}=0$. In the latter case, each trajectory does not have any effect on the updated $\mathbf{T}$ as depicted by (8) and (9). Expressed differently, the model does not build any history for the user, hence the prediction is independent of the mobility history. It is worth mentioning that the evaluation is performed without a training data set. Since the prediction is dependent on the historical trajecotry, the accuracy in the $1^{\text {st }}$ day is roughly $0 \%$ irrespective of the $R_{d}$ value. As a result, the average accuracy in Fig. 5 does not reach $100 \%$.

With $10 \%$ random days, the average prediction accuracy is $\approx 86 \%$ for $R_{d}$ in the range $[0.2,0.6]$. However, the accuracy decreases to $\approx 80 \%$ when $R_{d}>0.6$. Since the user performs some random movements, a high dependency on the most recent trajecotry slightly decreases the prediction accuracy. A similar behaviour can be observed in the $20 \%$ and the $30 \%$ random data scenarios where the prediction accuracy reaches a peak of $75 \%$ and $68 \%$, respectively, when $R_{d}$ in the range $[0.2,0.4]$ and decreases at larger values of $R_{d}$. In other words, reducing $R_{d}$ minimises the effect of random movements on the updated $\mathbf{T}$, but it increases the required observation period. Thus it can be said that $R_{d}$ is an important design parameter
TABLE I

Cost VALUES FOR HO SIGNALLING MESSAGES

\begin{tabular}{c|c}
\hline Cost description & Value \\
\hline \hline Transmission cost between DBSs over X2 & 5 \\
\hline Transmission cost between UE and DBS (include processing) & 6.5 \\
\hline Transmission cost between DBS and MME & 5 \\
\hline Processing cost at DBS & 4 \\
\hline Processing cost at MME & 5 \\
\hline Processing cost at S-GW & 5 \\
\hline Cost to detach from the source DBS and access the target DBS & 12 \\
\hline
\end{tabular}

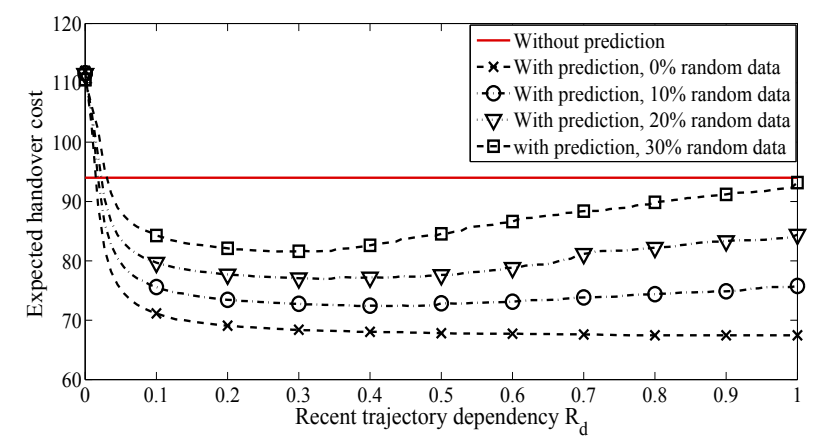

Fig. 6. HO signalling cost with and without mobility prediction

that can be tuned for each user to reach a particular accuracy target.

\section{B. Handover signalling cost}

Here we evaluate the potential benefits of the proposed mobility prediction scheme in terms of $\mathrm{HO}$ signalling cost. For simplicity, we follow [17] by assuming that the transmission cost for different messages between the same sourcedestination pair is the same irrespective of the message size. Similarly, the processing cost for different messages at the same node is constant. In addition, we assume that the mobility management entity (MME) and the serving gateway (S-GW) are located in the same location, thus the transmission delay between these nodes is negligible. Notice that the MME/S-GW transmission delay may be significant in vertical or inter-RAT ${ }^{4}$ HOs, however this case is not considered in this paper. Table I provides the cost values which are based on the feasibility study reported in [15] for the intra-LTE X2 HO procedure.

Fig. 6 provides the $\mathrm{HO}$ signalling cost for the non-predictive and the predictive $\mathrm{HO}$ procedures, where the mobility profiles of Section IV-A are considered for the predictive HO case. Comparing Fig. 5 with Fig. 6 indicates that the $R_{d}$ region that offers the peak accuracy also offers the minimum $\mathrm{HO}$ signalling cost. For the regular user scenario (i.e., $0 \%$ random data), the predictive $\mathrm{HO}$ signalling cost is $29 \%$ less than the non-predictive $\mathrm{HO}$ cost when $R_{d} \geq 0.3$. This can be traced to the high prediction accuracy in this region (see Fig. 5) which is translated into a reduction in the $\mathrm{HO}$ cost, i.e., most of the HOs are correctly predicted and hence the procedure for predictive $\mathrm{HO}$ with correct prediction is triggered in most

${ }^{4}$ Radio Access Technology 


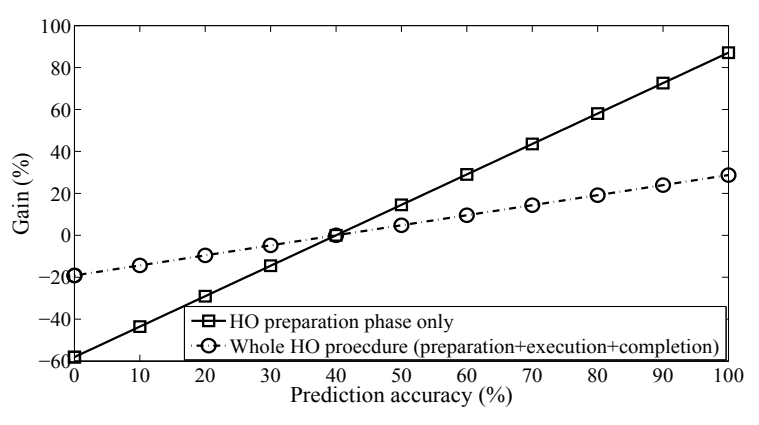

Fig. 7. Prediction accuracy vs predictive HO gains w.r.t. the traditional nonpredictive $\mathrm{HO}$

cases. It can be noticed that the predictive HO cost increases rapidly when $R_{d}<0.1$ and exceeds the non-predictive $\mathrm{HO}$ cost as $R_{d}$ reaches 0 . This can be traced to the low prediction accuracy in this region as shown in Fig 5.

With $10 \%, 20 \%$ and $30 \%$ random data and considering the peak accuracy region of each case, the predictive $\mathrm{HO}$ cost is roughly $23 \%, 18 \%$, and $13 \%$, respectively, less than the nonpredictive $\mathrm{HO}$ cost. It can be seen that the minimum signalling cost offered by the predictive $\mathrm{HO}$ biases towards small values of $R_{d}$ as the random data increases and vice versa. This can be linked to Fig. 5 where the peak accuracy region biases towards small $R_{d}$ values as the random data increases and vice versa, excluding the range $0 \leq R_{d} \leq 0.1$ where the learning process and the updated transition matrix are almost independent of the mobility history.

To generalise, Fig. 7 shows the prediction accuracy vs the predictive $\mathrm{HO}$ gain in terms of signalling cost reduction w.r.t. the non-predictive $\mathrm{HO}$, where a positive (negative) gain means a reduction (an increase) in the signalling cost. As can be seen, the predictive $\mathrm{HO}$ reduces the signalling cost provided that the prediction accuracy is higher than $40 \%$. As depicted by Fig. 7, a higher prediction accuracy results into a higher gain which can reach up to $30 \%$ with $A_{p}=100 \%$. However, when $A_{p}<40 \%$ the predictive $\mathrm{HO}$ increases the signalling cost by up to $20 \%$ as compared with the non-predictive $\mathrm{HO}$. Thus it can be concluded that the predictive $\mathrm{HO}$ management strategies require a lower bound for the prediction accuracy in order to be effective in reducing the signalling cost.

\section{CONCLUSION}

In this paper, we developed a mobility prediction model that can be used in predictive $\mathrm{HO}$ management strategies. A trajecotry dependency parameter is proposed to control the model's reaction to random and less frequent movements. The LTE X2 HO procedure is considered as a benchmark for the conventional non-predictive HO strategy. In addition, the standard procedure is adapted to the predictive scenario in order to evaluate its signalling cost. The latter is modelled as a function of the transmission and the processing delays for each message involved in the HO process. Numerical results show that the predictive $\mathrm{HO}$ strategy can significantly reduce the signalling cost. Moreover, the prediction accuracy is found to be an important parameter and a lower bound for the accuracy is required to minimise the signalling overhead. Although an appropriate setting of $R_{d}$ could improve the prediction accuracy for random users, a hybrid prediction technique consisting of mobility history along with dynamic movement prediction (e.g., speed and probable angle of movement) may give better results.

\section{ACKNOWLEDGEMENT}

This work was made possible by NPRP grant No. 5-10472437 from the Qatar National Research Fund (a member of The Qatar Foundation). The statements made herein are solely the responsibility of the authors. We would like to acknowledge the support of the University of Surrey 5GIC members for this work.

\section{REFERENCES}

[1] J. Andrews, H. Claussen, M. Dohler, S. Rangan, and M. Reed, "Femtocells: Past, present, and future," IEEE J. Sel. Areas Commun., vol. 30, no. 3, pp. 497-508, April 2012.

[2] J. Hoydis, M. Kobayashi, and M. Debbah, "Green small-cell networks," IEEE Veh. Technol. Mag., vol. 6, no. 1, pp. 37-43, March 2011.

[3] Nokia Siemens Networks, "2020: Beyond 4G, Radio Evolution for the Gigabit Experience," White Paper, August 2011. [Online]. Available: http://nsn.com/file/15036/ 2020-beyond-4g-radio-evolution-for-the-gigabit-experience

[4] 3GPP, "Study on small cell enhancements for E-UTRA and E-UTRAN: Higher layer aspects," Tech. Rep., December 2013, 3GPP TR 36.842 version 12.0.0 Release 12. [Online]. Available: http://www.3gpp.org/DynaReport/36842.htm

[5] H. Ishii, Y. Kishiyama, and H. Takahashi, "A novel architecture for LTEB :C-plane/U-plane split and phantom cell concept," in Proc. of IEEE Globecom Workshops, December 2012, pp. 624-630.

[6] I. Godor et al., "EARTH project deliverable D3.3: Final report on green network technologies," July 2012. [Online]. Available: http://bscw.ict-earth.eu/pub/bscw.cgi/d70472/EARTH_WP3_D3.3.pdf

[7] S. Liu, J. Wu, C. H. Koh, and V. Lau, "A $25 \mathrm{~Gb} / \mathrm{s}(/ \mathrm{km} 2)$ urban wireless network beyond IMT-advanced," IEEE Commun. Mag., vol. 49, no. 2, pp. 122-129, February 2011.

[8] 3GPP, Nokia Siemens Networks, "Mobility statistics for macro and small cell dual-connectivity cases," Tech. Rep., 3GPP TSG-RAN WG2 Meeting , Chicago, USA, 15-19 April 2013. [Online]. Available: http://www.3gpp.org/DynaReport/TDocExMtg--R2-81b--30048.htm

[9] A. Capone, A. Fonseca dos Santos, I. Filippini, and B. Gloss, "Looking beyond green cellular networks," in Proc. of 9th Annual Conference on Wireless On-demand Network Systems and Services, January 2012, pp. $127-130$

[10] J. R. Norris, Markov chains. Cambridge university press, 1998.

[11] S. Gambs, M.-O. Killijian, and M. N. del Prado Cortez, "Next place prediction using mobility markov chains," in Proc. of the 1st ACM Workshop on Measurement, Privacy, and Mobility, 2012.

[12] D. Katsaros and Y. Manolopoulos, "Prediction in wireless networks by markov chains," IEEE Wireless Commun., vol. 16, no. 2, pp. 56-64, April 2009.

[13] N. Amirrudin, S. Ariffin, N. Malik, and N. Ghazali, "User's mobility history-based mobility prediction in LTE femtocells network," in Proc. of IEEE International RF and Microwave Conference, December 2013, pp. $105-110$

[14] H. Abu-Ghazaleh and A. Alfa, "Application of mobility prediction in wireless networks using markov renewal theory," IEEE Trans. Veh. Technol., vol. 59, no. 2, pp. 788-802, February 2010.

[15] 3GPP, "Feasibility study for evolved Universal Terrestrial Radio Access (UTRA) and Universal Terrestrial Radio Access Network (UTRAN)," Tech. Rep., October 2012, 3GPP TR 25.912 version 11.0.0 Release 11. [Online]. Available: http://www.3gpp.org/DynaReport/25912.htm

[16] J. Ho and I. Akyildiz, "Local anchor scheme for reducing signaling costs in personal communications networks," IEEE/ACM Trans. Netw., vol. 4, no. 5, pp. 709-725, October 1996

[17] L. Wang, Y. Zhang, and Z. Wei, "Mobility management schemes at radio network layer for LTE femtocells," in Proc. of IEEE 69th Vehicular Technology Conference VTC Spring, April 2009. 